\section{P5.040 INTEGRATION OF GONORRHOEA AND CHLAMYDIA SELF-COLLECTION SERVICE WITHIN AN EXISTING REPRODUCTIVE HEALTH PROGRAMME IN KAMPALA, UGANDA}

doi:10.1136/sextrans-2013-051184.1084

'S M Mitchell, ${ }^{2 M}$ Sekikubo, ${ }^{2} \mathrm{C}$ Biryabarema, ${ }^{2} \mathrm{~J}$ Byamugisha, ${ }^{3} \mathrm{M}$ Steinberg, ${ }^{4} \mathrm{~J}$ Christilaw, ${ }^{1,4} \mathrm{D}$ M Money, ${ }^{1,5} \mathrm{G}$ S Ogilvie. 'University of British Columbia, Vancouver, BC, Canada; ${ }^{2}$ Makerere University, Kampala, Uganda; ${ }^{3}$ Simon Fraser University, Vancouver, BC, Canada; ${ }^{4}$ British Columbia Womens Hospital, Vancouver, BC, Canada; ${ }^{5}$ British Columbia Center for Disease Control, Vancouver, BC, Canada

Background Sub-Saharan Africa has the highest rates of curable sexually transmitted infections (STIs) globally with the greatest morbidity due to Neisseria gonorrhoea and Chlamydia trachomatis falling on women. In response to a demand for comprehensive reproductive health screening, we proposed that providing additional $N$. gonorrhoea and C. trachomatis testing within a cervical cancer screening programme involving self swabbing for HPV, would be acceptable and effective.

Methods As part of a cervical cancer screening project in Kisenyi, a densely populated urban community in Kampala, Uganda with low socio-economic-indicators, women aged 30 to 69 were offered $N$. gonorrhoea and C. trachomatis testing using self-collected swabs. Women were recruited in each sub-division by local health workers, after informed consent, a brief demographic and attitudes survey was completed and the method of swab collection was explained. Specimens were transported for PCR analysis within Kampala. Participants were contacted by mobile phone and asked to attend the local health clinic to receive appropriate treatment if found to be positive. Results Out of 206 women approached, 203 provided a self collected swab for analysis. Twenty-six women (13\%) were found to have $C$. trachomatis, $2(<1 \%)$ were positive for $N$. gonorrhoea and one participant was co-infected. Of the women infected, $76 \%$ were successfully contacted and of these $62 \%$ attended follow-up to receive appropriate treatment. Women reporting no condom use in the last month accounted for $93 \%$ of those with C. trachomatis while use of the oral contraceptive pill was not associated with higher rates of either infection. The self-reported HIV positivity rate was $9.5 \%$.

Discussion The acceptance and uptake of testing for common STIs in this urban sub-Saharan environment was very positive. Due to the high burden of disease inferred by these easily treated infections, further integration of appropriate screening should be incorporated into existing reproductive health programmes.

\section{P5.041 TB TREATMENT FOR HIV POSITIVE PREGNANT WOMEN: CHALLENGES TO SCREENING AND DIAGNOSIS}

doi:10.1136/sextrans-2013-051184.1085

'E du Plessis, 'S Y Shaw, ${ }^{2} M$ Gichuhi, ${ }^{2} J$ Kimani, 'L Gelmon, 'L Gelmon, 'L S Avery. 'University of Manitoba, Winnipeg, MB, Canada; 'University of Nairobi, Nairobi, Kenya

Background According to Kenya's PMTCT guidelines, all HIV positive women who present for antenatal care should be tested for TB.

Methods HIV-positive, pregnant women were recruited from two maternity hospitals in Nairobi, Kenya. The results presented here are based on surveys completed at baseline as well as 48 hour follow up. This data was collected from 505 women as part of a study on the use of mobile technology in PMTCT programmes. Questionnaires included questions on socio-economic characteristics, history of current and previous pregnancies, knowledge of PMTCT, TB screening and treatment and the use of Nevirapine. Chi-square tests and multivariable logistic regression were used to assess statistically significant associations between variables of interest and TB screening. Results Overall screening for TB in our sample was $10.3 \%$ with no significant difference between the two hospitals $(11.4 \%$ versus
8.4\%). Analysis also revealed no significant difference between groups based on sociodemographic status (including age, education, marital status and income) or based on the number of antenatal visits or gestational age at first presentation.

Conclusion Reportedly, $80 \%$ of TB patients are given access to HIV testing and a further $27 \%$ of those who test pest positive are placed on ART. TB screening for pregnant women seem to be offered less regularly, however, with only $10 \%$ of women screened. In our sample, the lack of significant difference in screening between facility, by sociodemographic characteristics or by when they access services seems to suggest suboptimal TB screening in pregnant women is a systemic issue.

\section{P5.042 OUALITY ASSURANCE IN VISUAL INSPECTION OF THE CERVIX - THE SOUTH AFRICAN EXPERIENCE}

doi:10.1136/sextrans-2013-051184.1086

1,2C Firnhaber, ${ }^{3} \mathrm{~L}$ Mao, ${ }^{4} \mathrm{D}$ A Lewis, ${ }^{2} \mathrm{~B}$ Goeieman, ${ }^{1} \mathrm{~A}$ Swarts, ${ }^{2} \mathrm{M}$ Faesen, ${ }^{2} \mathrm{~S}$ Levin, ${ }^{2} \mathrm{~N}$ Rakhombe, ${ }^{2}$ S Williams, ' J S Smith. 'University of Witwatersrand, Johannesburg, South Africa; ${ }^{2}$ Right to Care, Johannesburg, South Africa; ${ }^{3}$ University of North Carolina, Chapel Hill, NC, United States; ${ }^{4}$ National Institute of Communicable Diseases, Johannesburg, South Africa

Background Invasive cervical cancer is the most common cancer is Sub-Saharan Africa partly due to lack of effective screening programmes and the HIV epidemic. The baseline analysis of a South Africa study comparing three cervical cancer screening methods, i.e. cytology, visual inspection with acetic acid (VIA) and HPV testing in HIV-infected women, has been completed and quality assurance of VIA interpretation was evaluated.

Methods HIV-infected women, aged 18-65, were recruited from Johannesburg, South Africa and VIA performed with $5 \%$ acetic acid. Nurses received a two-week training on the VIA procedure. VIA interpretation by the nurse and recording of a digital image was performed in real time. The study physician reviewed weekly the digital imaging and recorded their own interpretation of the VIA appearance. Women with a positive VIA and $25 \%$ of women with a negative VIA had confirmatory colposcopic biopsy. Sensitivity and specificity for VIA interpretation for cervical intraepithelial neoplasia (CIN)-2 + for both the nurse and the doctor were compared retrospectively.

Results 1202 HIV infected women with a median age of 37 years and CD4 of 394 cells $/ \mathrm{mm}^{3}$ participated. $45 \%$ of the women had a positive VIA result. Sensitivity and specificity for VIA to determine CIN $2+$, as performed by the nurse and doctor, was $65.4 \%$ (59.7$71.1) / 68.5 \%(65.3-71.7)$ and $75.5 \%(70.5-80.4) / 68.1(65.0-71.3)$ respectively. There was no statistical difference in the VIA readings comparing the first 600 VIA interpretation to the final 593 VIA readings [nurse $(p=0.613)$ or doctors $(p=0.624)$ ]. Evaluating the first 300 VIA to the final 300 results was also similar ( $p=0.505$ for nurse; $p=0.802$ for doctors).

Conclusion Throughout the study there was no statistical difference in the sensitivity in interpreting VIA. These results show that after two weeks of training, nurses were consistent in their VIA readings and could accurately predict $65.4 \%$ of CIN $2+$.

\section{P5.043 EARLY EVALUATION OF THE ISEAN HIVOS PROGRAM'S HIV-AIDS BCC ONLINE INITIATIVE FOR MSM AND TG COMMUNITIES IN SOUTH EAST ASIA}

doi:10.1136/sextrans-2013-051184.1087

\section{A Lesmana. Hivos, Jakarta, Indonesia}

Online BCC Outreach Intervention was developed by the ISEAN Hivos Program to reach the hidden MSMs and TGs populations in Indonesia, Malaysia, Philippines and Timor Leste. The initiative 